\section{Cane sign: Sciatic Neuropathy Appearance in Magnetic Resonance Imaging}

Berin Inan, $\mathrm{MD}^{1}$; Can Ebru Bekircan-Kurt, $\mathrm{MD}^{1}$; Onur Akca, MD²; Sevim Erdem-Ozdamar, $\mathrm{MD}^{1}$; Ersin Tan, $\mathrm{MD}^{1}$

\section{${ }^{1}$ Department of Neurology, Hacettepe University Faculty of Medicine, Ankara, Turkey \\ ${ }^{2}$ Integra Medical Imaging Center, Ankara, Turkey}

An-eighteen-year-old male patient presented with slowly progressive foot drop on the left for two years. He was also suffering from a sharp pain radiating down the left posterolateral limb. There was no history of trauma. Neurological examination revealed weakness in knee flexion, foot eversion and inversion (4/5 according to the Medical Research Council (MRC) motor scale), foot dorsiflexion ( $1 / 5$, according to the MRC), and hypoesthesia in the lateral aspect of the leg, dorsum and lateral part of the foot on the left. Left Achilles reflex was also absent.

Magnetic resonance imaging revealed diffuse thickening and intense contrast enhancement of the left sciatic nerve, starting from the lumbar and sacral nerve roots, extending $10 \mathrm{~cm}$ distal to the popliteal fossa. This enlarged appearance of the sciatic nerve looks like a "cane"; thus, we decided to use the term "cane sign." Sciatic neuropathy, perineuroma, schwannoma, and malignant peripheral nerve sheathtumorswere consideredintheradiological differential diagnosis. Perineuroma and schwannoma were unlikely because the involved segment was too long. Similarly, long segment and cylindrical involvement were atypical features for a neurofibroma. Moreover, since it must have already invaded the surrounding soft tissues within two years, the diagnosis of malignant nerve sheath tumor was also avoided (Figure 1). Electroneuromyography showed a proximal lesion between the popliteal fossa and lumbosacral plexus in all branches of the left sciatic nerve, characterized by severe and chronic axonal loss. Laboratory work-up including autoimmune and infectious markers were unremarkable. With these findings, we suggested that idiopathic sciatic neuropathy was the most likely diagnosis. Five days of $1000 \mathrm{mg}$ intravenous methylprednisolone followed by a tapering course of oral methylprednisolone were started. As the patient did not benefit from this treatment, 3 months later, a trial of intravenous immunoglobulin (IVIg) 0.4 $\mathrm{g} / \mathrm{kg} /$ day for 5 days followed by monthly infusions was given. Unfortunately, improvement of muscle strength was insignificant, but his pain was relieved by gabapentinoids. Follow-up electrophysiological study depicted severe and chronic axonal loss of all branches of left sciatic nerve with minimal regeneration / reinnervation findings.

Sciatic neuropathy is the second most common neuropathy of the lower limbs and a frequent cause of foot drop. ${ }^{1}$ Drop-foot may be associated with many clinical syndromes, so electrophysiological examinations

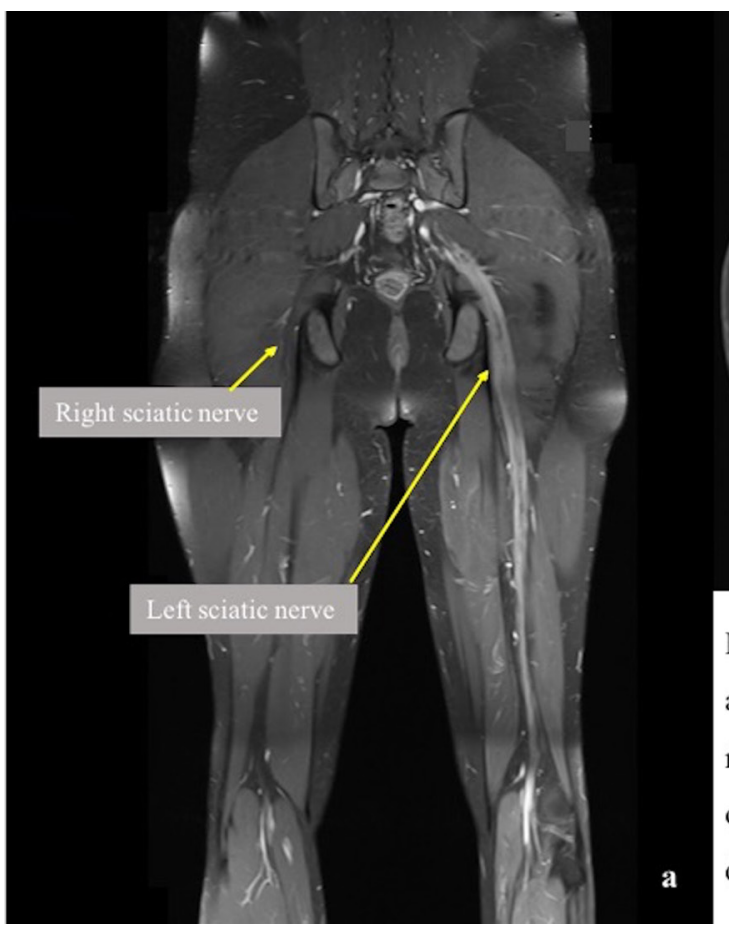

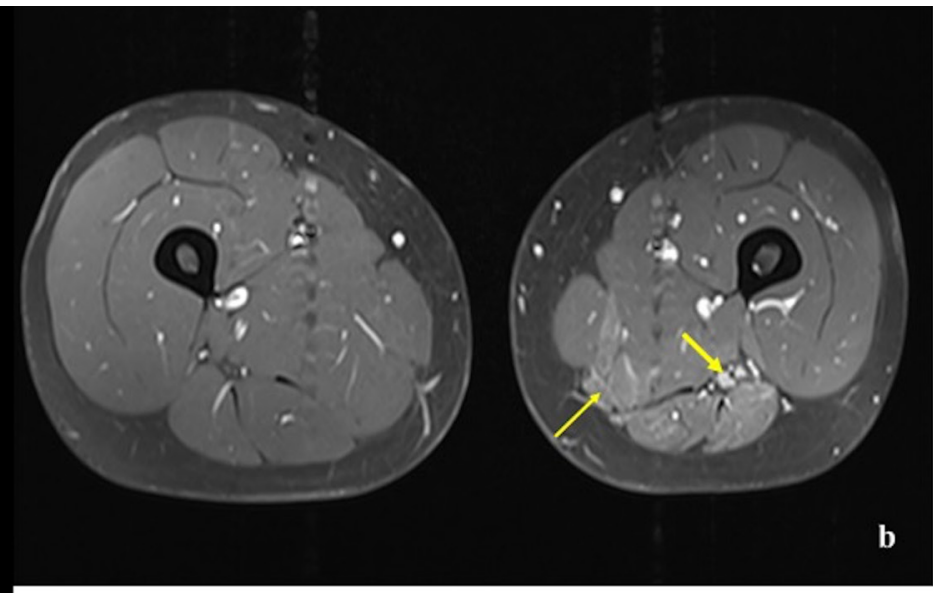

Figure 1 Lumbosacral magnetic resonance imaging. Coronal (a) and axial (b) gadolinium-enhanced T1-weighted, fat-saturated magnetic resonance images show diffuse thickening and enhancement of the left sciatic nerve. Contrast-enhancement and volume loss of denervated muscles are prominent in the axial image (b). 
and imaging studies, as well as clinical and neurological examination findings, are essential in the differential diagnosis.1, 2 Trauma, surgery, injections, infections, inflammation, vascular events, or tumors can cause sciatic neuropathy and up to $16 \%$ of cases, it can be idiopathic. ${ }^{1,3}$ If any, treatment of the underlying cause, immunosuppressive and supportive treatments, and physical therapy applications can be beneficial. ${ }^{1}$ Unfortunately, in our case, who was presented with demonstrative magnetic resonance imaging findings, delayed medical treatment initiation caused an insufficient clinical response.

\section{References}

${ }^{1}$ Distad BJ, Weiss MD. Clinical and electrodiagnostic features of sciatic neuropathies. Phys Med Rehabil Clin N Am 2013;24:107-120.

${ }^{2}$ Cherian RP, Li Y. Clinical and Electrodiagnostic Features Of Nontraumatic Sciatic Neuropathy. Muscle Nerve 2019;59:309-314.

${ }^{3}$ Yuen EC, Olney RK, So YT. Sciatic neuropathy: clinical and prognostic features in 73 patients. Neurology 1994;44:1669-1674. 